\title{
Integral Equations Satisfied by Lamé-Wangerin Functions
}

\author{
By C. G. LAMBE \\ (Received 5th March 1951. Read 2nd June 1951.)
}

Summary.

Integral equations are obtained with nuclei $(1-z t / a)^{2 n}$ and $(z-t)^{2 n}$ which are satisfied by characteristic solutions of the transformed Lamé-Wangerin equation of order $n$, and each of the two characteristic solutions is expressed in terms of the other by a contour integral.

\section{.. Introduction.}

Lamé-Wangerin functions are the solutions of Lamés differential equation of order $n$ when $n$ is half of an odd integer. If $n=m+\frac{1}{2}$, where $m$ is an integer, Lame's equation in algebraic form is

$$
\begin{aligned}
4 x(x-1)(x-a) \frac{d^{2} u}{d x^{2}}+2\left\{3 x^{2}-2(a+1) x+a\right\} \frac{d u}{d x} \\
-\left\{\left(m+\frac{1}{2}\right)\left(m+\frac{3}{2}\right) x+h\right\} u=0
\end{aligned}
$$

and there is no loss of generality in taking the finite singularities as 0,1 and $a$.

$$
\begin{aligned}
& \text { Writing } \quad x=\left(z^{2}-a\right)^{4} / 4 z(z-1)(z-a) \\
& u(x)=\{z(z-1)(z-a)\}^{(2 m+1) / 4} v(z),
\end{aligned}
$$

we have the transformed equation considered by Halphen and others: ${ }^{\mathrm{s}}$

$$
\begin{aligned}
z(z-1) & (z-a) \frac{d^{2} v}{d z^{2}}-m\left\{3 z^{2}-2(a+1) z+a\right\} \frac{d v}{d z} \\
& +\left\{\left(m+\frac{1}{2}\right)\left(m+\frac{\hat{z}}{2}\right) z-h-\left(m+\frac{1}{2}\right)^{2}(a+1)\right\} v=0 .
\end{aligned}
$$

If $h$ has one of a set of $m+1$ characteristic values this equation has two solutions which are polynomials in $z$. I shall denote them by

$$
\begin{aligned}
& v_{1}(z)=\sum_{==0}^{m} c_{\nu} a^{m+1-\nu} z^{\nu}, \\
& v_{2}(z)=\sum_{=-0}^{m} c_{\nu} z^{2 m+1-\nu},
\end{aligned}
$$

1 G. H. Halphen, Traité des Fonctions Elliptiques (Paris, 1888), t.2, p. 471. Whittaker and Watson, Modern Analysis (Cambridge, 1920, 3rd ed.), §\$ 23-7. 
the coefficients $c_{v}$ being the same in the two solutions and $c_{0}=1$. Substitution of either of these solutions in (1.2) leads to the recurrence relations

$$
\begin{aligned}
(\nu-m)(\nu+1) c_{\nu+1}- & \left\{h+\left(m+\frac{1}{2}-\nu\right)^{2}(a+1)\right\} c_{\nu} \\
& +a(m+1-v)(2 m+2-\nu) c_{\nu-1}=0
\end{aligned}
$$

for $v=0,1, \ldots, m$.

The first $m$ relations determine the coefficients $c_{1}$ to $c_{m}$, the coefficient $c_{v}$ being a polynomial of degree $\nu$ in $h$; and putting $\nu=m$ we have

$$
-\{h+(a+1) / 4\} c_{m}+(m+2) a c_{m-1}=0 .
$$

The expression in (1.5) is a polynomial of degree $m+1$ in $h$ determining $m+1$ characteristic values of $h$ which are real and distinct.

Wo have also

$$
v_{2}(z)=\frac{z^{2 m+1}}{a^{m+1}} v_{1}(a / z), \quad v_{1}(z)=\frac{z^{2 m+1}}{a^{m+1}} v_{2}(a / z) .
$$

2. Types of integral equation.

The differential equation (1.2) in Riemannian form is

$$
v(z)=P\left\{\begin{array}{cccc}
0 & 1 & a & \infty \\
0 & 0 & 0 & -m \\
m+1 & m+1 & m+1 & -2 m-1
\end{array}\right\} .
$$

A general theorem ${ }^{1}$ on integral equations associated with differential equations of this type shows that characteristic and certain other solutions of (1.2) satisfy an integral equation whose nucleus is the hypergeometric function

$$
P\left\{\begin{array}{ccc}
0 & 1 & \infty \\
0 & 0 & -m \\
m+1 & 2 m+1 & -2 m-1
\end{array} z t / a\right\} .
$$

We may take this function as being $\left(1^{*}-z t / a\right)^{2 m+1}$ and consider integrals of the type

$$
u(z)=\frac{\lambda}{2 \pi i} \int_{c}\left(1-\frac{z t}{a}\right)^{2 m+1}\{t(t-1)(t-a)\}^{m+1}
$$

where $C$ is a closed curve encircling one or more of the singularities $t=0, t=1, t=a$.

1 C. G. Lambé and D. R. Ward, Quart. J. of Math. (Oxford), 5 (1934), 81 and A. Erdélyi, Quart. J. Math. (Oxford), 13 (1942), 107. 
The general theorem shows that if $v(z)$ is a solution of (1.2) corresponding to a characteristic value $h_{\nu}$ of $h$, then $u(z)$ is also a solution of (1.2) for the same value of $h$.

From the relations (1.6) it follows that if $z$ is replaced by $a / z$,

$$
u(a j z)=a^{m+1} z^{-2 m-1} w(z),
$$

where $w(z)$ is a solution of (1.2). Hence integrals of the type

$$
w(z)=\frac{\lambda}{2 \pi i} \int_{C}(z-t)^{2 m+1} \frac{v(t) d t}{\{t(t-1)} \frac{(t-a)\}^{m}}{(t+1}
$$

are also solutions of (1.2).

3. Contours enclosing one singularity.

If $C$ is a contour enclosing only the singularity $t=0$, the value of the integral (2.2) is the residue at $t=0$. Hence $u(z)$ must be of degree $m$ in $z$ and therefore must be a multiple of $v_{1}(z)$.

If $C$ encloses only the singularity $t=1$, then, since powers of $(t-1)$ higher than the $m^{t h}$ in the expansion of

$$
(1-z t / a)^{2 m+1}=\{(1-z / a)-(t-1) z / a\}^{2 m+1}
$$

give zero residue at $t=1$, it follows that $(z-a)^{m+1}$ is a factor of $u(z)$ and hence

$$
u(z)=A\left\{v_{1}(a) v_{2}(z)-v_{2}(a) v_{1}(z)\right\}
$$

where $A$ is a constant.

If $C$ encloses the singularity $t=a$, then, since

$$
(1-z t / a)^{2 m+1}=\{(1-z)-(t-a) z / a\}^{2 m+1},
$$

it follows that $(z-1)^{m+1}$ is a factor of $u(z)$ and hence

$$
u(z)=B\left\{v_{1}(1) v_{2}(z)-v_{2}(1) v_{1}(z)\right\}
$$

where $B$ is a constant.

Hence for these contours, if the equation (2.2) is to be an integral equation the function $v(t)$ in the integrand must be the same function of $t$ as the corresponding $u(z)$ is of $z$. Using the relations (1.6) we have, therefore, solutions of the integral equation.

$$
v(z)=\frac{\lambda}{2 \pi i} \int_{C}(1-z t / a)^{2 m+1} \frac{v(t) d t}{\{t(t-1)(t-a)\}^{m+1}}
$$

for contours encircling one singularity only as:

$$
\text { around } \begin{aligned}
t & =0, V_{0}(z)=v_{2}(0) v_{2}(z)-v_{1}(0) v_{1}(z), \\
, \quad t & =1, V_{1}(z)=v_{2}(1) v_{2}(z)-v_{1}(1) v_{1}(z), \\
, \quad t & =a, V_{a}(z)=v_{2}(a) v_{2}(z)-v_{1}(a) v_{1}(z) .
\end{aligned}
$$


4. Value of $\lambda$.

The constant $\lambda$ involves the characteristic value of $h$ for any solution of the integral equation (3.1) and may be evaluated in terms of the coefficient $c_{m}$ of $z^{m}$ in $v_{1}(z)$. Let $\lambda_{0}, \lambda_{1}, \lambda_{a}$ denote the values of $\lambda$ for the three contours considered.

Substituting $V_{0}(z)$ in (3.1) and comparing coefficients of $z^{m}$, we have

$$
\begin{aligned}
-v_{1}(0) a^{\frac{1}{2}} c_{m} & =\frac{\lambda_{0}}{2 \pi i} \int_{(0+)} \frac{(2 m+1) !}{m !(m+1) !}\left(-\frac{1}{a}\right)^{m} \frac{V_{0}(t) d t}{t(t-1)^{m+1}(t-a)^{m+1}} \\
& =\lambda_{0} \frac{(2 m+1) !}{m !(m+1) !}\left(-\frac{1}{a}\right)^{n} \frac{V_{0}(0)}{a^{m+1}} \\
V_{0}(0) & =-v_{1}^{2}(0)=-a^{m+1} v_{1}(0),
\end{aligned}
$$

and hence

$$
\lambda_{0}=(-)^{m} \frac{\Gamma\left(\frac{1}{2}\right)(m+1) !}{2^{2 m+1} \Gamma\left(m+\frac{3}{2}\right)} a^{m+1} c_{m} .
$$

We have seen that $V_{1}(z)$ contains the factor $(z-a)^{m+1}$, and hence $V_{1}(t) /(t-a)^{m+1}$ is a polynomial in $(t-1)$ of which the first term is $v_{2}(1)(t-1)^{m}$. Substituting $V_{1}(t)$ in (3.1) and comparing coefficients of $z^{m+1}$, we have

$$
\begin{aligned}
v_{2}(1) c_{m} & =\frac{\lambda_{1}}{2 \pi i} \int_{(1+)} \frac{(2 m+1) !}{m !(m+1) !}\left(-\frac{1}{a}\right)^{m+1} v_{2} \frac{(1)(t-1)^{m}+\cdots}{(t-1)^{m+1}} d t . \\
& =\lambda_{1} \frac{(2 m+1) !}{m !(m+1) !}\left(-\frac{1}{a}\right)^{m+1} v_{2}(1) .
\end{aligned}
$$

Hence $\lambda_{1}=-\lambda_{0}$.

Similarly, substituting $V_{a}(z)$ in (3.1) and comparing coefficients of $z^{m+1}$, we find that $\lambda_{\alpha}=-\lambda_{0}$.

\section{Therefore}

$$
\lambda_{a}=\lambda_{1}=-\lambda_{0}=(-)^{m+1} \frac{\Gamma\left(\frac{1}{2}\right)(m+1)^{!}}{2^{2 m+1} L^{\prime}\left(m+\frac{3}{2}\right)} a^{m+1} c_{m} .
$$

5. Contour enclosing two singularities.

Since $V_{1}(t)$ has the factor $(t-a)^{m+1}$ and $V_{a}(t)$ the factor $(t-1)^{m+1}$, we have

$$
\begin{aligned}
& A V_{1}(z)=\frac{\lambda_{1}}{2 \pi i} \int_{(1+1}\left(1-\frac{z t}{a}\right)^{2 m+1} \frac{A V_{1}(t)+B V_{2}(t)}{\{t(t-1)(t-a)\}^{m+1}} d t \\
& B V_{a}(z)=\frac{\lambda_{1}}{2 \pi i} \int_{(a+)}\left(1-\frac{z t}{a}\right)^{2 m+1} \frac{A V_{1}(t)+B V_{2}(t)}{\{t(t-1)(t-a)\}^{m+1}} d t,
\end{aligned}
$$

where $A$ and $B$ are any constants. 
Integral Equations Satisfied by Lamé-Wangerin Functions 75

Hence

$A V_{1}(z)+B V_{a}(z)=\frac{\lambda_{1}}{2 \pi i} \int_{(1+, a+)}\left(1-\frac{z t}{a}\right)^{2 m+1} \frac{A V_{1}(t)+B V_{2}(t)}{\{t(t-1)(t-a)\}^{m+1}} d t$

It follows that any solution of the differential equation (1.2) when $h$ has a characteristic value satisfies the integral equation

$$
v(z)=\frac{\lambda_{1}}{2 \pi i} \int_{(1+, a+)}\left(1-\frac{z t}{a}\right)^{2 m+1}\left\{\frac{v(t) d t}{i(t-1)(t-a)\}^{m+1}} d t .\right.
$$

6. Nucleus $(z-t)^{2 m+1}$.

Substituting $a / z$ for $z$ in (3.1), we have

$$
z^{2 m+1} v(a / z)=\lambda \int_{C}(z-t)^{2 m+1} \frac{v(t) d t}{\{t(t-1)(t-a)\}^{m+1}} .
$$

Hence for contours around one singularity we have, using (1.6),

$$
\begin{aligned}
a^{m+1} v_{2}(z) & =\frac{\lambda_{0}}{2 \pi i} \int_{(0+)}(z-t)^{2 m+1} \overline{v_{1}(t) d t} \\
-V_{a}(z) & =\frac{\lambda_{1}}{2 \pi i} \int_{(1+)}(z-t)^{2 m+1} \frac{V_{1}(t) d t}{\{t(t-1)(t-a)\}^{m+1}} \\
-a^{2 m+1} V_{1}(z) & =\frac{\lambda_{a}}{2 \pi i} \int_{(a+1)}(z-t)^{2 m+1} \frac{V_{a}(t) d t}{\{t(t-1)(t-a)\}^{m+1}} .
\end{aligned}
$$

Combining these results as before, we have

$$
\begin{aligned}
& v_{2}(z)=a^{-m-\frac{1}{2}} \frac{\lambda_{1}}{2 \pi i} \int_{(1+\cdot a+)}(z-t)^{2 m+1} \frac{v_{1}(t) d t}{\left\{t \left(t-\frac{1)(t-a)\}^{m+1}}{(t-1)}\right.\right.} \lambda_{2 \pi i}^{\lambda_{1}} \int_{(1+, a+)}(z-t)^{2 m+1} \frac{v_{2}(t) d t}{\{t(t-1)(t-a)\}^{m+1}} .
\end{aligned}
$$

Hence the solution of the integral equation

$$
v(z)=\frac{\mu}{2 \pi i} \int_{(1+, a+)}(z-t)^{2 m+1} \frac{v(t) d t}{\{t(t-1)(t-a)\}^{m+1}}
$$

is $\quad v(z)=v_{2}(z) \pm v_{1}(z)$

and

$$
\mu= \pm(-)^{m+1} \frac{\Gamma\left(\frac{1}{2}\right)(m+1) !}{2^{2 m+1} \Gamma\left(m+\frac{3}{2}\right)} a^{\frac{1}{3}} c_{m} \text {. }
$$

Military College of Soience, SHRIVENHAM. 